\title{
Mandibular Implants: A Retrospective Single Private Surgical Practice Study
}

\author{
J Collum ${ }^{1 *}$, B Robinson ${ }^{2}$, P Sambrook ${ }^{3}$, A. Goss ${ }^{4}$ and A. Lynham ${ }^{5}$
}

${ }^{1}$ Registrar - Oral and Maxillofacial Surgery, Royal Brisbane and Women's Hospital, Queensland, Australia ${ }^{2}$ Oral and Maxillofacial Surgeon, in private practice, Adelaide, Australia

${ }^{3}$ Oral and Maxillofacial Surgery Unit, The University of Adelaide and private practice, Adelaide, Australia

${ }^{4}$ Oral \& Maxillofacial Surgery Unit, The University of Adelaide, Adelaide, Australia

${ }^{5}$ Oral and Maxillofacial Unit, Royal Brisbane and Women's Hospital, Queensland, Australia

\section{Abstract}

Background: Dental implants have revolutionised restorative dental care but this is complex surgical and prosthodontic treatment. Excellent results have been reported in many institutional studies with a small but acceptable failure rate. It is largely unknown which of these results translate directly into a private practice setting.

Methods: All patients who have dental implants placed in the maxilla by a single oral and maxillofacial surgeon in private practice in over a 3 year period were included in this study. All implants were placed in a two stage approach.

All the data was collected through retrospective review of patient files and radiographic assessment. Patient data included full demographics, referral source, type and site of implants placed, and adjunctive procedures.

The outcome including any complications in the first phase between placement and uncovering were recorded. The restorative phase was performed by a variety of general dentists and prosthodontists, with patients being followed up in surgical outpatients post final prostheses being issued.

In all cases the individual who referred the patient and performed the reconstruction had been present at the time of surgical implantation. The outcome, including any complications in the prosthodontic reconstruction phase was recorded.

This data was recorded on a standardised data sheet and maintained and analysed using SPSS (Statistical Package for the Social Sciences, version 9.05, Chicago, IL). Where two different factors were compared, the $95 \%$ confidence interval $(\mathrm{Cl})$ of the difference between the survival rates was calculated. A difference was considered statistically significant on a $5 \%$ level when this confidence level did not contain 0 .

Results: 179 mandibular implants were placed in 57 patients. On review at three years 166 (93\%) were successful with 13 implant failures. Of the implants which failed, nine of them failed in the first 6 months, and none after 18 months.

There were a number of trends which did not reach statistical significance. Failures were more common in the following circumstances; in the posterior mandible (11 of 13), with older mark II \& III version implants (12 of 13) and implants shorter than $8 \mathrm{~mm}$. There was no correlation to medical conditions or smoking. An incidental finding was a correlation between those who smoked and had heart disease. $(P<0.005)$

Keywords: Implants; Mandible; Mandibular complications

\section{Introduction}

Dental implants have revolutionised restorative dentistry and become common place. However failure may occur which can be painful, expensive and disappointing to both the patient and clinicians.

Implant failure has been associated with a number of risk factors such as decreased bone quality and volume, location within the jaw, implant mobility and unfavourable loading [1-5]. In some patient factors including some medical conditions such as diabetes and smoking [6-8], implant failure may occur before prosthetic fixture placement (early loss) or after (late failures) [4,5]. In the mandible and maxilla implant failure rates of $7 \%$ have been reported over a 20 year period [9], with the majority of failures (4.2\%) occurring prior to prosthodontic rehabilitation. Implant failure has been reported to range between $1.5-21 \%[3,10,11]$.

Implants placed in the mandible have been reported as having more favourable success rates than that of the maxilla [2,12]. This has been related to a comparative lower bone density of the maxilla than the mandible. Within the mandible it has been shown that success rates for the posterior region are significantly less than anteriorly [12].
This has been attributed to a decreased bone height and density from anterior to posterior.

Recommendations have been made that implant length should be of at least $8 \mathrm{~mm}$ and placed within sound bone $[10,13,14]$ and not within $2 \mathrm{~mm}$ of a vital nerve structure [15]. In the posterior mandible the inferior alveolar canal encasing the inferior alveolar nerve (IAN) must be considered. In atrophic mandibles implant placement may be contraindicated due to proximity of the IAN, or transposition techniques may be considered Computed tomography (CT scan) is

*Corresponding author: Justin Collum, Oral Maxillofacial Surgery Unit, Roya Brisbane and Womens Hospital, Queensland 4029, Australia, Tel: (07) 36367681 Fax: (07) 36367627; E-mail: jgcollum@gmail.com

Received February 13, 2012; Accepted March 21, 2012; Published March 23 2012

Citation: Collum J, Robinson B, Sambrook P, Goss A, Lynham A (2012) Mandibular Implants: A Retrospective Single Private Surgical Practice Study. Dentistry 2:126 doi:10.4172/2161-1122.1000126

Copyright: ( 2012 Collum J, et al. This is an open-access article distributed unde the terms of the Creative Commons Attribution License, which permits unrestricted use, distribution, and reproduction in any medium, provided the original author and source are credited. 
valuable in the comprehensive assessment of implant sites in particular in the atrophic posterior mandible [16].

It has been reported that the main reasons for early implant failure can be attributed to infection, anatomical conditions, surgical trauma and lack of operator surgical implant experience $[1,2,5,11,17]$. Most late implant losses relate to bone quality, volume and implant loading [18].

Most publications however related to multicentre academic institutional studies. Few studies relate to private referral practice situations [19].

The purpose of this study was to examine a retrospective, consecutive series of one brand of implants placed by a single surgeon in a private practice setting. Particular emphasis was placed on the small number of implants which failed which were investigated in detail.

\section{Methods and Materials}

\section{Patient and anatomical factors}

A total of 60 private patients who received 190 endosseous implants in the mandible in a 3 year period, with or without bone augmentation, were included in this study. This consisted of 29 male and 31 female patients whose age ranged from 15 to 78 years with a mean age of 52.4 years. Three patients were lost at follow up (11 implants), and thus excluded from the study. Two were females and one was male, all were non-smokers and had a mean age of 57.8 years. There were no other exclusions within this study. Thus 57 patients and 179 implants remained in the study.

Patient information was collected via case notes and radiographs. Pre-operative radiographs including orthopantomographs and peri apical films which were screened for any evidence of mandibular pathology and used to assess the height of the mandibular bone. Bone morphology was assessed according to Cawood and Howells classification [20]. The patients' medical history and smoking habits was recorded. All patients were reviewed for up to 3 years.

\section{Implant procedure}

All implant related operative procedures and bone augmentations were conducted by the same oral and maxillofacial surgeon. The prosthetic rehabilitation phase was performed by a variety of general dentists and specialist prosthodontists in Adelaide. All were in attendance with the surgeon at the time of implant placement.

Bone augmentation was performed where there was insufficient bone to facilitate an endosseous implant, or subsequent to post operative bone loss. This consisted of either autogenously corticocancellous bone grafts, or allograft including products such as biooss $^{\circledR}$, bio-gran ${ }^{\circledR}$, and peri-glass ${ }^{\circledR}$.

In cases where there was proximity to the IAN, CT imaging was used. Upon surgical identification, the neurovascular bundle was protected as no IAN transposition procedures were performed. Pre and post-operative sensation was assessed and recorded.

In all procedures, original Branemark System components were used. Of the total or 179 implants that were placed, these consisting of Mk 11, Mk 111, Mk lV and Ti unite surface types (Figure 1). Both single and two stage implant procedures were performed. The two stage implants were allowed an osseointegration period of 4-6 months prior to placement of healing abutments. In all cases prophylactic intravenous antibiotics were administered (Penicillin $n=55$, Clindamycin $n=2$ ). Antibiotics were continued for 5 days with appropriate oral analgesia.

\section{Post-operative evaluation}

The prosthetic phase of treatment was undertaken by the referring dentist or specialist prosthodontist 6-11 weeks after placement of healing abutments. All patients in the second stage were prosthodontically restored with a range of fixed prostheses, over dentures, partial bridges or crowns.

Following implantation peri-implant bone height was evaluated clinically and through the use postoperative radiographs. An implant was considered as 'failed' if one or more of the following criteria were met; clinical and/or radiographic evidence of loss of bone height; post-operative infection; peri apical radiolucency associated with the implant, or implant mobility.

A further clinical audit was performed on the patients in this study at 4 years after the main study thus giving a 5-8 year follow up.

\section{Statistical evaluation}

Statistical evaluation was done using SPSS (Statistical Package for the Social Sciences, version 9.05, Chicago, IL). Parametric, nonparametric and descriptive statistical methods were used to evaluate statistical significance between patient and implant sample groups, involving student t-test, chi-squared test and Pearson's test for correlation respectively. In all test the $95 \%$ confidence level was calculated.

\section{Results}

\section{Patient related factors}

Overall 166 of the 179 (93\%) implants were successfully integrated, dental reconstructed and functioning well on completion of the study. Thirteen implants in 11 patients failed.

Anatomical location: The distribution of 179 implants and their location in the mandible are presented in Figure 1. Eleven failures occurred in the posterior mandible (85\%), with 5 were in the molar region and 6 in the premolar region. Two (15\%) failures occurred in the anterior mandible.

Preoperative bone height: The preoperative bone height is presented in Figure 2. The majority of implants were placed either immediately post extraction (Cawood class 2, $\mathrm{n}=53,29 \%$ ) or in patients with adequate height and width of the alveolus (Cawood class $3 \mathrm{n}=77,42 \%)$. Implant sites with atrophic alveolar bone consisted of Cawood class 4, $(n=45,25 \%)$ and Cawood class 5, $(n=5,3 \%)$. Implant failure occurred in 6 class 3 sites (6.5\% of class 3 sites) and 7 class 4 sites

\section{Distribution of implant location and associated} failure

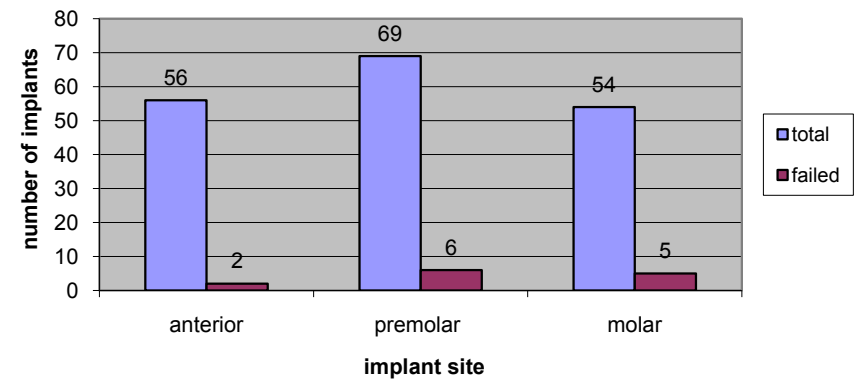

Figure 1: Distribution of implant location and associate failure. 
(14\%of class 4 sites). Significant differences between preoperative bone height and implant failure were not found.

Bone loss: Marginal bone loss was noted post-operatively at 6 month intervals up to three years (Figure 3). The majority of bone loss was within the first 6 months of implant placement and of that group, 9 implants failed. A total of 21 out of 179 implant sites had marginal bone loss at 6 months with 2 implant sites having substantial bone loss. At 12 months there were 5 sites with marginal bone loss, and at 18 months there were 3 sites with marginal bone loss with no implant failures. No bone loss or implant failure was seen in 18-36 months. Thus there was a tendency for failed implants sites to present with bone loss during the first 6 months of placement. This was not statistically significant.

Gender and age: The total patient sample consisted of 28 males (49\%) and 29 females (51\%). Of this sample, 6 of 28 (21\%) males and 5 of $29(17 \%)$ females had implant failure (Figure 4).

There was a higher mean age in the implant failure group. Mean age for implant failure was $\mathbf{5 7 . 6}$ years as compared to 51.5 years for implant survival (Figure 5). This was not statistically significant.

Bimaxillary cases: Of the 57 patients, 53 were bimaxillary cases (10 patients/12 implants failed, 19\%) and 4 were mandibular implant cases only (1 patients / 1 implant failed, 25\%). There was no significant difference in failure rate between single or double jaw placements.

Full/partial edentulism: Of the 57 patients, 43 were partially edentulous with 9 patients experiencing implant failure (21\%). There were 14 fully edentulous patients with 2 patients experiencing implant

\section{Distribution of mandibular pre-operative bone height} and associated failure

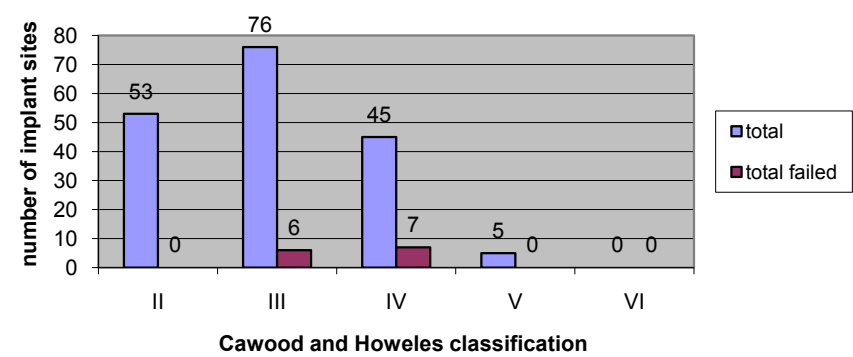

Figure 2: Distribution of mandibular pre-operative bone height and associated failure.

\section{Bone Loss and associated failure vs time}

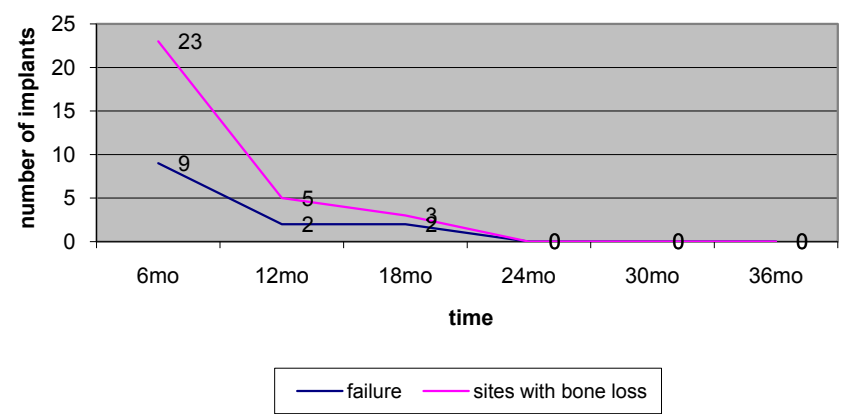

Figure 3: Bone Loss and associated failure vs time.

\section{Distribution of patient gender and associated failure}

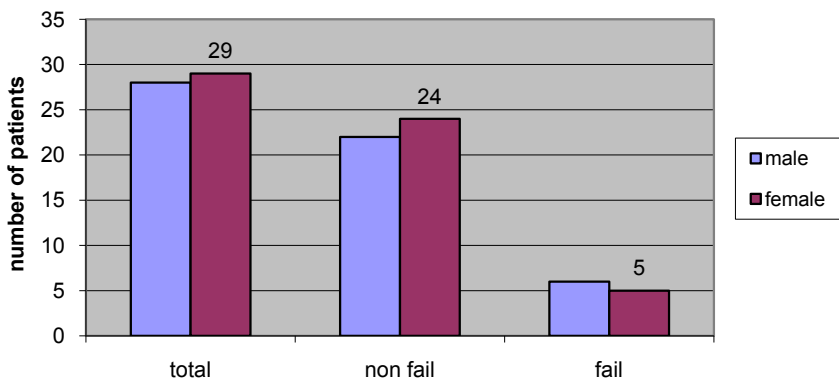

Figure 4: Distribution of patient gender and associated failure.

Age Distribution and associated implant failure

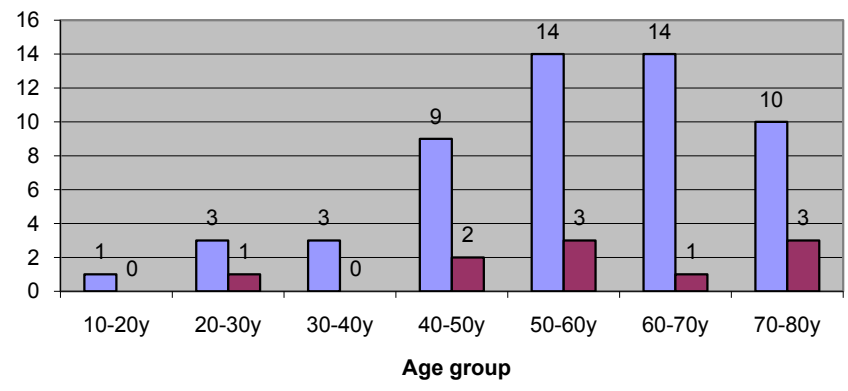

Figure 5: Age distribution and associated implant failure.

failure (14\%). There was no significant difference in failure rate between the two samples.

Osteoporosis and diabetes: In this study there was 1 type two diabetic and 1 patient with known osteoporosis. Neither of these patients experienced implant failure.

\section{Implant related factors}

Thirteen implants failed and were removed during stage 2 abutment connection procedures or during the healing period. The reasons for implant removal included implant mobility $(n=6)$, bone loss $(n=2)$ and infection $(n=5)$. The 13 failed implants were in 11 patient cases. This gave a success rate of $93 \%$ (92.7) for implants placed in the mandible. Of all 13 implants lost, 9 were lost within 6 months (69\%), 2 were lost within in 12 months (15.4\%) and 2 were lost between 12-18 months (15.4\%). No further implant loss was reported 18 months post implant placement.

Suprastructure: The various suprastructure used include 24 single unit crowns, 18 partial fixed bridges, 3 over dentures and 12 patients for full fixed prostheses in the mandible. Of the failure occurred association with crowns (8.33\%) partial fixed bridges (4.22\%) and full fixed prostheses (1.8\%). No failures occurred with the over dentures. Although there was a higher rate of failure with single unit crowns, this was not statistically significant.

Implant type: Most failures occurred with the earlier implant systems. Mark II (7 fail) and mark III (5 fail) represent $92 \%$ of the failed implants $(n=13)$. There were no failures in the mark IV system and there was only one failed implant in the Ti Unite system (Figure 
6). With regard to single and 2 stage implant systems there were 36 single stage implants of which 6 failed (17\%) and 143 2-stage implants of which 7 (4\%) failed.

Shorter fixtures had a greater frequency of implant failure compared to longer implants $(>15 \mathrm{~mm})$ (Figure 7$)$. Statistical significance was approached between implant length and failure $(\mathrm{P}=07)$.

Grafts: Bone augmentation procedures were performed in 17 patients. This consisted of autogenous cortico-cancellous grafts $(n=7)$ and synthetic bone substitutes $(n=7)$ and synthetic bone substitutes $(\mathrm{n}=10)$ : Bio-oss $=7^{\circledR}$, Bio-gran $=2^{\circledR}$ and Perioglass $\left.{ }^{\circledR}=1\right)$. Of these, 5 patients had 7 implant which failure. Augmentation procedures were initiated at second stage surgery when there was evidence of marginal bone loss. In these patients, the implants were removed and replaced in conjunction with graft placement. None subsequently failed.

\section{Surgical related factors}

Post operative surgical complications involved 4 patients which presented with post operative infection. These patients had to 6 implant failures (6 of 13). Treatment was curettage and antibiotic therapy.

No long term sensory disturbance occurred where implants were placed in proximity to the inferior alveolar nerve.

The clinical audit at 4 years post main study showed no important changes in the findings.

\section{Discussion}

This study shows that there was a high success rate when Branemark implants were placed in the mandible by a single oral and maxillofacial

\section{Distribution of Branemark implant types and} associated failure

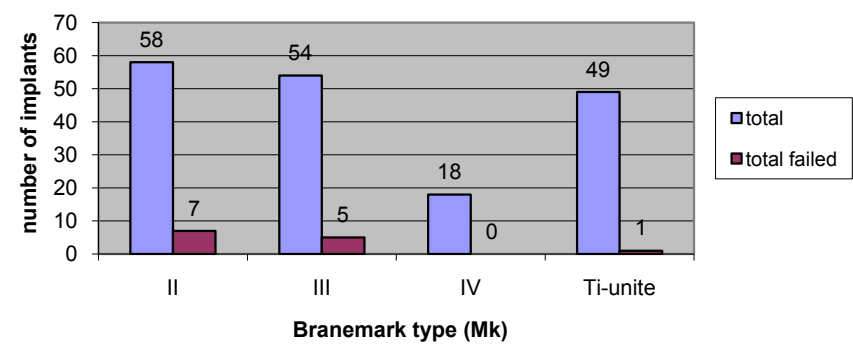

Figure 6: Distribution of branemark implant types and associated failure.

\section{Distribution of implant length and associated failure}

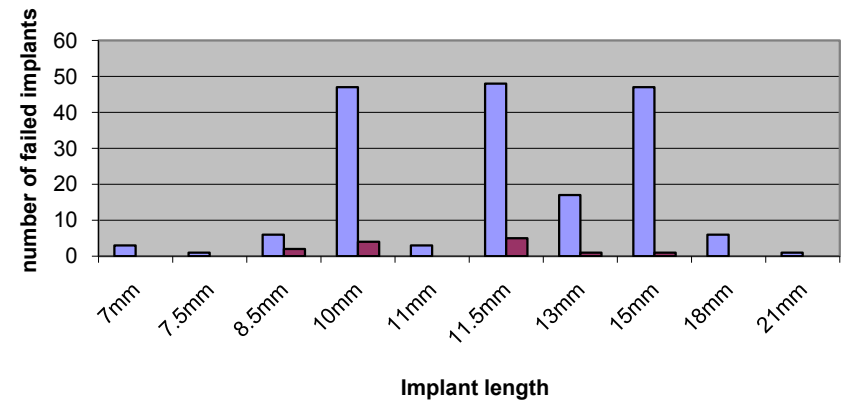

Figure 7: Distribution of implant length and associated failure. surgeon working in private practice. The success rate for mandibular dental implants in this study support the current literature that a high success rate can be achieved with Branemark implants used for the rehabilitation of the oral cavity in partial and full edentulism [14]. With the exclusion of patient dropout, dental implant success in the mandible was $93 \%$. This is a slightly lower success rate thank the $96 \%$ rate presented van Steenberghe et al. [14].

Possible reasons for this slightly lower success rate may be attributed to the design of the study, being retrospective, with no exclusion criteria for patients and thus inclusion of all dental implant failures prior to stage 2 surgery.

The limitations of this study relate to a relatively low sample size with statistical significance for many of the results being unable to be calculated. This was because low sample sizes of groups with nominal data (eg smoking failures: 3 implants) which violated one of the hypothesis of the chi squared test which had a minimum sample size of 5. This has limited this report to descriptive results only, with inferential results being rejected as non-significant due to the low sample sizes. With a larger population group representing greater sample sizes, statistical significance could then be calculated. This has been performed in such academic multicenter studies as van Steenberghe et al. [14] and thus is a limitation in the design of this study which was focussed on one private oral maxillofacial surgeon's implant cases. This however is the real world circumstance.

Data collection was limited to patient records and reliance upon accurate case notes and radiographs. Thus potential bias and omission of information is a possibility and limits the validity of the results of this retrospective of this study.

All dental implant failures occurred within 18 months of placement, with $85 \%$ of these occurring within 12 months of placement. There appears to be a relationship between bone loss and implant failure during this first 6 month period with $70 \%$ of the failed implants occurring within this time. As successful osseointegration requires stable bone height during the healing phase, subsequent bone loss leads to sub-optimal conditions for integration and can be attributed to the substantial number of implants which failed during this period of bone loss. This study reported a higher rate (7\%) of early implant failure compared with Zarb and Elsubeihi [9] who reported a $4.2 \%$ failure rate.

Implant failure of the earlier implant systems Mk II (7) and III (5) represented $92 \%$ of all implant failures. There was only one failure in the Ti unite system, which occurred in a 47 year old male smoker. The implant was $10 \mathrm{~mm}$ in length and placed in the posterior mandible. There were no failures with the Mk IV system. This may be attributed to the larger samples of the earlier Mk II and III systems accounting for $63 \%(62.4 \%)$ of the patient sample. No significant difference between implant versions was found. In terms of single versus two stage implant placement there was no significant difference in failure, with single stage failure contributing to $54 \%$ (7 of 13 implants), and two stage contributing to $46 \%$ (6 of 13 implants) of implant failure.

Implants placed in the posterior region of the mandible (premolar and molar region) had a greater rate of failure $(85 \%$ of the failed implants) compared to the anterior region (15\%), although this correlation was not significant. This supports recent studies including Becker et al. [12] whereby a decrease in bone height and density in the posterior region was cited as the key factor.

With regards to implant length, a significant correlation was 
approached between length and failure, with increase in failure correlated with a decrease in implant length. This supports other studies in which a greater rate of failure was reported amongst shorter implants $[10,13,14]$ and recommended at least $8 \mathrm{~mm}$ length for successful osseointegration.

Using Cawood and Howell's [20] classification of mandibular bone type, there was no significant correlation between preoperative bone height and implant failure. However there was a higher failure rate in unsatisfactory bone type (class 4 ). Thus, it may be inferred that as atrophic bone loss in the mandible increases, there is an associated increased risk for non-integration of dental implants. The lower failure rate of satisfactory preoperative bone heights of class 5 bone types did not impair osseointegration in the mandible. There were no failures in the class 5 category, but this consisted of only 1 patient with 5 successful implants.

Age and gender did not relate to implant failure although there was no significant difference reported, although there was a slight increase in the mean age of patients with failed implants. This is in agreement with Dao et al. [21] who reported no correlation between patient age or gender and dental implant failure. The patient's medical state did not relate to implant failure, this may be attributed to the relative low sample size of patients who smoke, have diabetes or osteoporosis in this study. This is in contrast to many published studies showing an associated significant risk between such factors and implant failure [2230]. The relatively small sample sizes of such at-risk groups relates to case selection by the surgeon. All smokers were counselled about the increased risk of failure. There was a significant association between smoking and heart disease in this patient sample $(\mathrm{P}=0.005)$, which supports current medical opinion of the association between smoking and heart disease $[31,33]$.

There were no significant differences reported between partial and fully edentulous patients. This is in contrast to Esposito et al. [11] who reported that implants placement in partially dentate patients had a more favourable survival rate compared with those placed in fully edentulous patients. This disparity may be attributed to the poor representation of fully edentulous patients within the patient sample at only $25 \%$ of population sample.

No patient had mandibular nerve damage which shows the importance of careful case selection, use of CT scanning and a skilled and experienced surgeon.

This study fails to prove causality between any associated risk factors with implant failure in the mandible. As stated by Zarb and Elsubeihi [9] identification of causality usually requites randomized intervention studies which are difficult to design and perform. Thus ongoing clinical and experimental studies are needed to further elucidate potential causes of implant failure within the mandible.

Importantly this study shows similar results - single private practice surgeon working with multiple practitioners referring and performing the second stage reconstruction to multicentre institutional studies.

\section{References}

1. Kronström M, Svenson B, Hellman M, Persson GR (2001) Early implant failures in patients treated with Brånemark System titanium dental implants: a retrospective study. Int J Oral Maxillofac Implants 16: 201-207.

2. Friberg B, Jemt T, Lekholm U (1991) Early failures in 4,641 consecutively placed Brånemark dental implants: a study from stage 1 surgery to the connection of completed prostheses. Int J Oral Maxillofac Implants 6: 142-146.

3. Tinsley D, Watson CJ, Ogden AR (1999) A survey of U.K. centres on implant failures. J Oral Rehabil 26: 14-18.
4. Truhlar RS, Morris HF, Ochi S, Winkler S (1994) Second-stage failures related to bone quality in patients receiving endosseous dental implants: DICRG Interim Report No. 7. Dental Implant Clinical Research Group. Implant Dent 3: 252-255.

5. Duyck J, Naert I (1998) Failure of oral implants: aetiology, symptoms and influencing factors. Clin Oral Investig 2: 102-114.

6. Nordin BE (1987) The definition and diagnosis of osteoporosis. Calcif Tissue Int 40: 57-58.

7. Koch JP, Dunson B (1996) Factors affecting bone healing following implant surgery. J Oral Implantol 22: 7-11.

8. Cullen R (1998) The oral-burn syndrome and its effects on dental implants. $J$ Oral Implantol 24: 219-221.

9. Elsubeihi ES, Zarb GA (2002) Implant prosthodontics in medically challenged patients: the University of Toronto experience. J Can Dent Assoc 68: 103-108.

10. Friberg B, Jemt T, Lekholm U (1991) Early failures in 4,641 consecutively placed Brånemark dental implants: a study from stage 1 surgery to the connection of completed prostheses. Int J Oral Maxillofac Implants 6: 142-146.

11. Esposito M, Hirsch JM, Lekholm U, Thomsen P (1998) Biological factors contributing to failures of osseointegrated oral implants. Eur J Oral Sci 106: 527-551.

12. Becker W, Becker BE, Alsuwyed A, Al-Mubarak S (1999) Long-term evaluation of 282 implants in maxillary and mandibular molar positions: a prospective study. J Periodontol 70: 896-901.

13. Leckholm U (1992) The Branemark implant technique: a standard procedure under continuous development. Tissue Integration in Oral, Orthopaedic and Maxillofacial Reconstruction. Chicago.

14. van Steenberghe D, Lekholm U, Bolender C, Folmer T, Henry P, et al. (1990) Applicability of osseointegrated oral implants in the rehabilitation of partial edentulism: a prospective multicenter study on 558 fixtures. Int J Oral Maxillofac Implants 5: 272-281.

15. Nevins M, Mellonig JT (1998) Implant Therapy: Clinical Approaches and Evidence of Success. New York.

16. Wyatt CC, Pharoah MJ (1998) Imaging techniques and image interpretation for dental implant treatment. Int J Prosthodont 11: 442-452.

17. Preiskel HW, Tsolka P (1995) Treatment outcomes in implant therapy: the influence of surgical and prosthodontic experience. Int J Prosthodont 8: 273 279

18. Sennerby L, Roos J (1998) Surgical determinants of clinical success of osseointegrated oral implants: a review of the literature. Int J Prosthodont 11 : 408-420.

19. Walton JN, MacEntee MI (1997) A prospective study on the maintenance of implant prostheses in private practice. Int J Prosthodont 10: 453-458.

20. Cawood JI, Howell RA (1988) A classification of the edentulous jaws. Int J Oral Maxillofac Surg 17: 232-236.

21. Dao TT, Anderson JD, Zarb GA (1993) Is osteoporosis a risk factor for osseointegration of dental implants? Int J Oral Maxillofac Implants 8: 137-144.

22. Haas R, Haimböck W, Mailath G, Watzek G (1996) The relationship of smoking on peri-implant tissue: a retrospective study. J Prosthet Dent 76: 592-596.

23. Sennerby L, Roos J (1998) Surgical determinants of clinical success of osseointegrated oral implants: a review of the literature. Int J Prosthodont 11: 408-420.

24. Berglundh T, Lindhe J, Ericsson I, Marinello CP, Liljenberg B, et al. (1991) The soft tissue barrier at implants and teeth. Clin Oral Impl res 2: 81-90.

25. Berglundh T, Lindhe J, Marinello C, Ericsson I, Liljenberg B (1992) Soft tissue reactions to de novo plaque formation on implants and teeth. An experimental study in the dog. Clin Oral Impl Res 3: 1-8.

26. Lindhe J, Berglundh T, Ericsson I, Liljenberg B, Marinello C (1992) Experimenta breakdown of peri-implant and periodontal tissues. A study in the beagle dog Clin Oral Implants Res 3: 9-16.

27. The UK Smoking Epidemic: Deaths in 1995. Health Education Authority, 1998

28. Doll R, Peto R, Wheatley K, Gray R, Sutherland I (1994) Mortality in relation to smoking: 40 years' observations on male British doctors. BMJ 309: 901-911. 
Citation: Collum J, Robinson B, Sambrook P, Goss A, Lynham A (2012) Mandibular Implants: A Retrospective Single Private Surgical Practice Study. Dentistry 2:126. doi:10.4172/2161-1122.1000126

29. Cole CW, Hill GB, Farzad E, Bouchard A, Moher D, et al. (1993) Cigarette smoking and peripheral arterial occlusive disease. Surgery 114: 753-756.

30. Morrison A, Chiarot M, Kirby S (2002) Mental nerve function after inferior alveolar nerve transposition for placement of dental implants. J Can Dent Assoc 68: 46-50.
31. Bartling R, Freeman K, Kraut RA (1999) The incidence of altered sensation of the mental nerve after mandibular implant placement. J Oral Maxillofac Surg 57: $1408-1412$.

32. Morris HF, Ochi S, Winkler S (2000) Implant survival in patients with type 2 diabetes: placement to 36 months. Ann Periodontol 5: 157-165. 